\title{
Screening for Fabry disease and hereditary ATTR amyloidosis in idiopathic small
}

\section{fiber and mixed neuropathy}

Kristin Samuelsson, PhD, MD, ${ }^{1}$ Ana Radovic, MD, ${ }^{2}$ Rayomand Press, PhD, MD, ${ }^{1}$ Mari Auranen, PhD, MD ${ }^{3}$ Emil Ylikallio, $\mathrm{PhD}, \mathrm{MD},{ }^{3}$ Henna Tyynismaa, $\mathrm{PhD},{ }^{3}$ Mikko Kärppä, $\mathrm{PhD}, \mathrm{MD},{ }^{4}$ Matilda Veteläinen, MD,${ }^{4}$ Niina Peltola, MD,${ }^{5}$ Svein Ivar Mellgren, PhD, MD,${ }^{6}$ Åse Mygland, $\mathrm{PhD}, \mathrm{MD},{ }^{7}$ Chantal Tallaksen, $\mathrm{PhD}, \mathrm{MD},{ }^{8}$ Henning Andersen, $\mathrm{PhD}, \mathrm{MD},{ }^{9}$ Astrid Juhl Terkelsen, PhD, MD, ${ }^{9}$ Freja Fontain, MD, ${ }^{9}$ Aki Hietaharju, PhD, $\mathrm{MD}^{5}$

${ }^{1}$ Department of Clinical Neuroscience, Karolinska Institutet, Stockholm, Sweden ${ }^{2}$ Department of Neurology, Karolinska University Hospital, Stockholm, Sweden ${ }^{3}$ Research Programs Unit, Molecular Neurology, University of Helsinki, Helsinki, Finland ${ }^{4}$ Research Unit of Clinical Neuroscience, Neurology, University of Oulu, Oulu, Finland ${ }^{5}$ Department of Neurology, Tampere University Hospital and Faculty of Medical and Life Sciences, University of Tampere, Tampere, Finland

${ }^{6}$ Department of Clinical Medicine, UiT The Arctic University of Norway and University Hospital of North Norway, Department of Neurology, Troms $\emptyset$, Norway

${ }^{7}$ Department of Neurology, Sørlandet Hospital, Kristiansand, Norway

${ }^{8}$ Department of Neurology, Oslo University Hospital and Faculty of Medicine, University of Oslo, Oslo, Norway

${ }^{9}$ Department of Neurology, Aarhus University Hospital, Aarhus, Denmark been through the copyediting, typesetting, pagination and proofreading process, which may lead to differences between this version and the Version of Record. Please cite this article as doi: $10.1002 /$ mus. 26348 


\section{Acknowledgements}

We thank Dr. Ingela Nygren, Department of Neurology, University of Uppsala, Sweden for valuable help in recruitment of patients, and Riitta Lehtinen and Mariia Shcherbii from Research Programs Unit, Molecular Neurology, University of Helsinki for technical assistance. Funding for this Investigator Sponsored Study was provided by Sanofi Genzyme and Alnylam Pharmaceuticals. The funding sources had no involvement in study design, in the collection, analysis an interpretation of the data, in the writing of the report and the decision to submit the article for publication.

Number of words in abstract: 145

Number of words in manuscript: 1447

Corresponding author: Kristin Samuelsson, Department of Neurology, R54, Karolinska University Hospital, Huddinge, 14186 Stockholm, Sweden, kristin.samuelsson@ki.se Running title: Idiopathic polyneuropathy

\section{Ethical Publication Statement}

We confirm that we have read the Journal's position on issues involved in ethical publication and affirm that this report is consistent with those guidelines.

\section{Disclosure of Conflict of Interest}

Dr Aki Hietaharju has received personal fees and non-financial support from Sanofi Genzyme outside the submitted work. The remaining authors have no conflict of interest, besides the grants from Sanofi Genzyme and Alnylam Pharmaceuticals during the conduct of the study declared under acknowledgements. 


\section{Abstract}

Introduction: We aimed to explore the value of genetic screening for Fabry disease (FD) and hereditary ATTR amyloidosis in patients with idiopathic small fiber neuropathy (SFN) or mixed neuropathy in a clinical setting.

Methods: This was a Nordic multicenter study with 9 participating centers. Patients with idiopathic SFN or mixed neuropathy were included. Genetic sequencing of the TTR and GLA genes was performed.

Results: 172 patients were enrolled. Genetic screening was performed in 155 patients. No pathogenic mutations in the TTR gene were found. A single patient had a possible pathogenic variant, $\mathrm{R} 118 \mathrm{C}$, in the GLA gene, but the clinical investigation showed no firm signs of FD.

Discussion: Screening for hereditary ATTR amyloidosis and FD in patients with idiopathic SFN or mixed neuropathy without any additional disease-specific symptoms or clinical characteristics in a Nordic population appears to be of little value in a clinical setting.

\section{Keywords}

Fabry disease; genetic screening study; hereditary ATTR amyloidosis; idiopathic polyneuropathy; small fiber neuropathy 


\section{INTRODUCTION}

Polyneuropathy is a common disorder with an age-dependent prevalence of $1-3 \%$ in the general population and up to 6-7 \% in the elderly. ${ }^{1-2}$ The etiology often remains unclear, especially in patients with slowly progressive predominantly sensory axonal polyneuropathy and small fiber neuropathy $(\mathrm{SFN}){ }^{2-3}$

Two treatable, possibly underdiagnosed hereditary diseases, known to cause painful polyneuropathy with involvement of small fibers, are Fabry disease (FD) and hereditary ATTR (hATTR) amyloidosis. ${ }^{4,5}$ The results of previous screening studies of the two conditions have been inconsistent. ${ }^{6-10}$

In this study we aimed to further explore the value of genetic screening for FD and hATTR amyloidosis in patients with idiopathic SFN or mixed neuropathy (combined SFN and large fiber sensorimotor axonal polyneuropathy) in a clinical setting in the Nordic region.

\section{METHODS}

This retrospective and prospective multicenter study, performed during October 2015February 2017, involved 9 participating neurology departments from 4 different Nordic countries.

The study was carried out according to the Helsinki Declaration and was approved by the local ethical committee in each country. All participants received written and oral information about the study, as well as genetic counseling, prior to inclusion and gave their written informed consent upon entering the study. 
The inclusion criteria were age $\geq 18$ years, with a diagnosis of SFN, consisting of patients with a pure SFN or mixed neuropathy (combined SFN and large fiber sensorimotor axonal polyneuropathy) without any identified etiology. The diagnosis of SFN was confirmed by clinical examination, abnormal warm or cold quantitative sensory testing (TSA-II NeuroSensory Analyzer, Medoc Ltd, USA, or Thermotest Somedic AB Type I, Sweden) and/or skin biopsy. The exclusion criteria consisted of a previously-established diagnosis of FD or hATTR amyloidosis, previously performed genotyping for transthyretin (TTR) and/or alpha-galactosidase A (GLA) mutations, or other conditions known to cause SFN, such as diabetes, uremia, thyroid disease, vitamin deficiency, immune mediated neuropathies, alcohol abuse, neurotoxic drugs and infections.

In the retrospective part of the study patients were identified through diagnosis registers at each center and a subsequent review of medical records. If the SFN had been judged as idiopathic by the responsible neurologist at the time for SFN diagnosis, the patient was contacted by letter and invited to participate in the study. The patients were thoroughly interviewed to exclude possible SFN associated-conditions. No further etiological investigation was performed. If possible SFN-associated co-morbidities started after the timepoint of SFN diagnosis, a case-to case judgement regarding the possible association with SFN was performed. For the prospectively included patients the etiological investigation was performed according to each center's clinical routine. The evaluations were not standardized.

A blood sample was collected from all patients who met the inclusion- and exclusion criteria.

\section{Genotyping}

The TTR gene exons 1-4 and GLA gene exons 1-7 and part of intron 4 were Sanger sequenced. GLA copy number variation was determined by multiplex ligation-probe assay. 


\section{Statistics}

The Kruskal-Wallis multiple comparison test for non-parametric values was used for comparison between the countries. For comparison of binary data between countries Chisquare testing was used.

\section{RESULTS}

A total of 172 patients were enrolled. Seventeen were subsequently excluded (Fig. 1).

\section{Clinical Description}

Patient characteristics are shown in Table 1. The patients from Norway were significantly younger than the patients from the other countries, with a median age of 54 years. There was no significant difference regarding sex, duration of symptoms or the distribution between isolated SFN or mixed neuropathy between the different centers. All included patients from Denmark and Norway had pain, which significantly differed from the patients from Finland and Sweden, among whom $89.7 \%$ and $71.7 \%$ had pain, respectively. Approximately $60 \%$ of the included patients reported autonomic symptoms, the most common being gastrointestinal, hypo- or hyperhidrosis and orthostatic hypotension.

\section{Genetic results}

We found no pathogenic mutations in the TTR gene. In the GLA gene we found a single patient harboring an earlier described possible pathogenic variant, p.Arg118Cys, also referred to as $\mathrm{R} 118 \mathrm{C}$.

\section{Patient with a possible pathogenic R118C mutation in the GLA gene}

This 69-year old male from Sweden, had a 10 year history of slowly progressive, mildly painful isolated SFN with symptoms restricted to his feet. He had no family history of polyneuropathy. Dried blood spot showed normal $\alpha$-galactosidase $(2.6 \mu \mathrm{mol} / \mathrm{L} / \mathrm{h}$, reference 
value: $2.3-17)$ and lyso-globotriaosylceramide (lyso-Gb3) levels (1.2 ng/ml, cut off values 0.0-3.5). Further clinical investigation showed no typical FD deposits in the eyes and no typical skin changes. The MRI of the heart showed no hypertrophy and normal global function. The brain MRI showed no typical FD features. He had a mildly impaired kidney function (estimated glomerular filtration rate (eGFR) $55 \mathrm{~mL} / \mathrm{min} / 1.7$, reference value $>60$ $\mathrm{mL} / \mathrm{min} / 1.7$ ) probably due to a postrenal obstruction secondary to an earlier urinary tract malignancy.

\section{DISCUSSION}

In this multicenter study of 155 Nordic patients with idiopathic small fiber neuropathy and mixed neuropathy, no definite FD and hATTR amyloidosis cases were identified. This result is concordant with earlier screening studies for FD and hATTR amyloidosis in patients with idiopathic SFN. ${ }^{7-9}$

The idea of FD as an underdiagnosed disease in patients with symptoms from an isolated organ system arose when Spada et al published their screening study of newborns in Italy and found a high frequency of late-onset GLA mutations. ${ }^{11}$ Screening studies of patients with cryptogenic stroke, hypertrophic cardiac disease and isolated kidney dysfunction have similarly shown a higher frequency of FD than expected. ${ }^{12-14}$ The same hypothesis drove the German pilot study which identified one case of FD in a group of 24 patients with idiopathic SFN. ${ }^{6}$ However, this was not confirmed in a small controlled study of patients with idiopathic polyneuropathy, ${ }^{7}$ nor in a large genetic screening study of 440 Dutch patients with isolated SFN. ${ }^{8}$ In a recent Chinese study one case of FD was identified in 100 patients with idiopathic SFN. ${ }^{10}$ 
The variant R118C was first described as a possible pathogenic late-onset mutation by Spada et al. ${ }^{11}$ It has been considered pathogenic in patients with cryptogenic stroke and chronic kidney failure. ${ }^{12,14}$ In a large screening study of Dutch patients with SFN, the R118C variant was identified in one woman. She had normal biochemistry and showed no FD manifestations during 3 years of follow-up. ${ }^{8}$ Recently, a review of clinical, biochemical and histopathological data of 22 patients with the R118C variant was published. ${ }^{15}$ The patients had normal life time expectancy and no major organ complications, however some had angiokeratoma. The authors suggest the variant to be non-pathogenic or of low pathogenicity and not to warrant enzymatic treatment. ${ }^{15}$ However, in another recent publication, R118C was suggested to be a late-onset mutation. ${ }^{16}$ Our patient with R118C had no clinical manifestations of FD and his enzyme level was normal. With a slowly progressive isolated SFN, very mild kidney dysfunction probably due to postrenal obstruction, and negative biomarkers, there is no firm evidence supporting the diagnosis of FD in this patient.

Fabry disease has no specific geographic distribution, but a high prevalence and awareness of hATTR amyloidosis (predominantly the Val50Met type) is expected as a possible cause of SFN in endemic areas such as the north of Sweden. ${ }^{17}$ In non-endemic areas one could suspect hATTR amyloidosis to be underdiagnosed, though screening for TTR mutations in countries with more genetic diversity implies a higher probability of negative results. In this multicenter study with a Nordic cohort of patients with idiopathic polyneuropathy we found no new cases of hATTR amyloidosis, which is consistent with the findings in a small genetic screening study of patients with idiopathic SFN from the US. ${ }^{9}$ However, Hsu et al found 3 cases (Ala97Ser) of hATTR amyloidosis in a cohort of 100 Chinese patients with an idiopathic pure SFN. ${ }^{10}$ 
The major strength of this study is the multicenter approach including a large cohort of patients from different Nordic countries. However, due to the multicenter approach and retrospective design, the extent of etiological investigation of SFN varied between centers. By including only patients with a seemingly idiopathic SFN, we are aware that we may have ruled out patients with a known possible SFN-associated disease who might also have hATTR amyloidosis or FD However, since the Dutch study ncluded patients with other diseaseassociated etiologies of $\mathrm{SFN},{ }^{8}$ wider inclusion criteria probably would not have affected our results. Likewise, the exclusion of patients with previously-performed screening of the GLA and/or the TTR genes or known mutations in either of the genes introduced a bias with the risk of missing cases with either or both of the diseases.

Screening for hATTR amyloidosis and FD in patients with idiopathic SFN without any additional disease-specific symptoms or clinical characteristics in a Nordic population thus appears to be of little value in a clinical setting. Nevertheless, the divergent results in earlier genetic screening studies in patients with idiopathic SFN suggest that in some populations screening for these disorders might be worthwhile. ${ }^{6,10}$ 


\section{ABBREVIATIONS}

$\mathrm{FD}=$ Fabry Disease

$G L A=$ alpha-galactosidase A gene

hATTR = hereditary ATTR

$\mathrm{SFN}=$ small fiber neuropathy

$T T R=$ transthyretin gene 


\section{REFERENCES}

1. Hoffman EM, Staff NP, Robb JM, St. Sauver JL, Dyck PJ, Klein CJ. Impairments and comorbidities of polyneuropathy revealed by population-based analyses. Neurology 2015;84(16):1644-51.

2. Hanewinckel R, van Oijen M, Ikram MA, van Doorn PA. The epidemiology and risk factors of chronic polyneuropathy. Eur J of Epidemiol 2016;31(1):5-20.

3. Peters MJ, Bakkers M, Merkies IS, Hoeijmakers JG, van Raak EP, Faber CG. Incidence and prevalence of small-fiber neuropathy: a survey in the Netherlands. Neurology 2013;81(15):1356-60.

4. Ranieri M, Bedini G, Parati EA, Bersano A. Fabry Disease: Recognition, Diagnosis, and Treatment of Neurological Features. Curr Treat Options Neurol 2016;18(7):33.

5. Adams D, Suhr OB, Hund E, Obici L, Tournev I, Campistol JM, et al. First European consensus for diagnosis, management, and treatment of transthyretin familial amyloid polyneuropathy. Curr Opin Neurol 2016;29 Suppl 1:S14-26.

6. Tanislav C, Kaps M, Rolfs A, Böttcher T, Lackner K, Paschke E, et al. Frequency of Fabry disease in patients with small-fibre neuropathy of unknown aetiology: a pilot study. Eur $J$ Neurol 2011;18(4):631-6.

7. Samuelsson K, Kostulas K, Vrethem M, Rolfs A, Press R. Idiopathic small fiber neuropathy: phenotype, etiologies, and the search for fabry disease. J Clin Neurol 2014;10(2):108-18.

8. de Greef BT, Hoeijmakers JG, Wolters EE, Smeets HJ, van den Wijngaard A, Merkies IS, et al. No Fabry Disease in Patients Presenting with Isolated Small Fiber Neuropathy. PLoS One 2016;11(2):e0148316.

9. Levine TD, Bland RJ. Incidence of nonamyloidogenic mutations in the transthyretin gene in patients with autonomic and small fiber neuropathy. Muscle Nerve 2018;57(1):140-142. 
10. Hsu JL, Liao MF, Hsu HC, Weng YC, Lo AL, Chang KH, et al. A prospective, observational study of patients with uncommon distal symmetric painful small-fiber neuropathy. PLoS One 2017;12(9):e0183948

11. Spada M, Pagliardini S, Yasuda M, Tukel T, Thiagarajan G, Sakuraba H, et al. High incidence of later-onset fabry disease revealed by newborn screening. Am J Hum Genet 2006;79(1):31-40.

12. Baptista MV, Ferreira S, Pinho-E-Melo T, Carvalho M, Cruz VT, Carmona C, et al. Mutations of the GLA gene in young patients with stroke: the PORTYSTROKE study-screening genetic conditions in Portuguese young stroke patients. Stroke 2010;41(3):431-6. 13. Elliott P, Baker R, Pasquale F, Quarta G, Ebrahim H, Mehta AB, et al. Prevalence of Anderson-Fabry disease in patients with hypertrophic cardiomyopathy: the European Anderson-Fabry Disease survey. Heart 2011;97(23):1957-60.

14. Herrera J, Miranda CS. Prevalence of Fabry's disease within hemodialysis patients in Spain. Clin Nephrol 2014;81(2):112-20.

15. Ferreira S, Ortiz A, Germain DP, Viana-Baptista M, Caldeira-Gomes A, Camprecios M, et al. The alpha-galactosidase A p.Arg118Cys variant does not cause a Fabry disease phenotype: data from individual patients and family studies. Mol Genet Metab 2015;114(2):248-58.

16. Gonçalves MJ, Mourão AF, Martinho A, Simões O, Melo-Gomes J, Salgado M, et al. Genetic Screening of Mutations Associated with Fabry Disease in a Nationwide Cohort of Juvenile Idiopathic Arthritis Patients. Front Med(Lausanne) 2017;4:12.

17. Parman Y, Adams D, Obici L, Galán L, Guergueltcheva V, Suhr OB, et al. Sixty years of transthyretin familial amyloid polyneuropathy (TTR-FAP) in Europe: where are we now? A European network approach to defining the epidemiology and management patterns for TTRFAP. Curr Opin Neurol 2016;29 Suppl 1:S3-s13. 
Table 1. Demographics and clinical characteristics of included patients

Included patients $n=155$

\begin{tabular}{ccc}
\hline Mean & Median & Range \\
\hline 60.9 & 63 & $21-86$
\end{tabular}

Age (years)

9.7

8

$0.5-40$

Duration of symptoms (years)

49

Female sex $(\%)$

64.5

Isolated SFN (\%)

Mixed neuropathy* (\%)

89

Neuropathic pain (\%)

73.5

QST performed (\%)

63.2

Skin biopsy performed $\uparrow(\%)$

GLA, alpha-galactosidase A gene; QST, quantitative sensory testing; SFN, small fiber neuropathy; TTR, transthyretin gene.

* Based on nerve conduction studies or in 2 cases where nerve conduction studies were

lacking, clinical examination indicating large fiber involvement.

$†$ Skin biopsy was performed in Denmark, Norway and Finland only. 
Figure legend.

\section{Figure 1. Flowchart of included and excluded patients in each country}

The exclusions were due to:

${ }^{a}$ Still's disease, not SFN phenotype and hypothyroidism

${ }^{\mathrm{b}}$ Active ulcerative colitis, hypothyroidism diagnosis coincidental with start of SFN symptoms, earlier screened GLA mutation, alcohol abuse

${ }^{c}$ Coeliac disease, systemic connective tissue disorder

${ }^{\mathrm{d}}$ Diabetes mellitus less than 2 years after onset of SFN, rheumatic disorder, Sjögren's syndrome, not SFN phenotype, active thyroid disease (Graves' disease), polyneuropathy associated with hematologic conditions (MGUS and cold agglutinin antibodies), alcohol abuse (two patients)
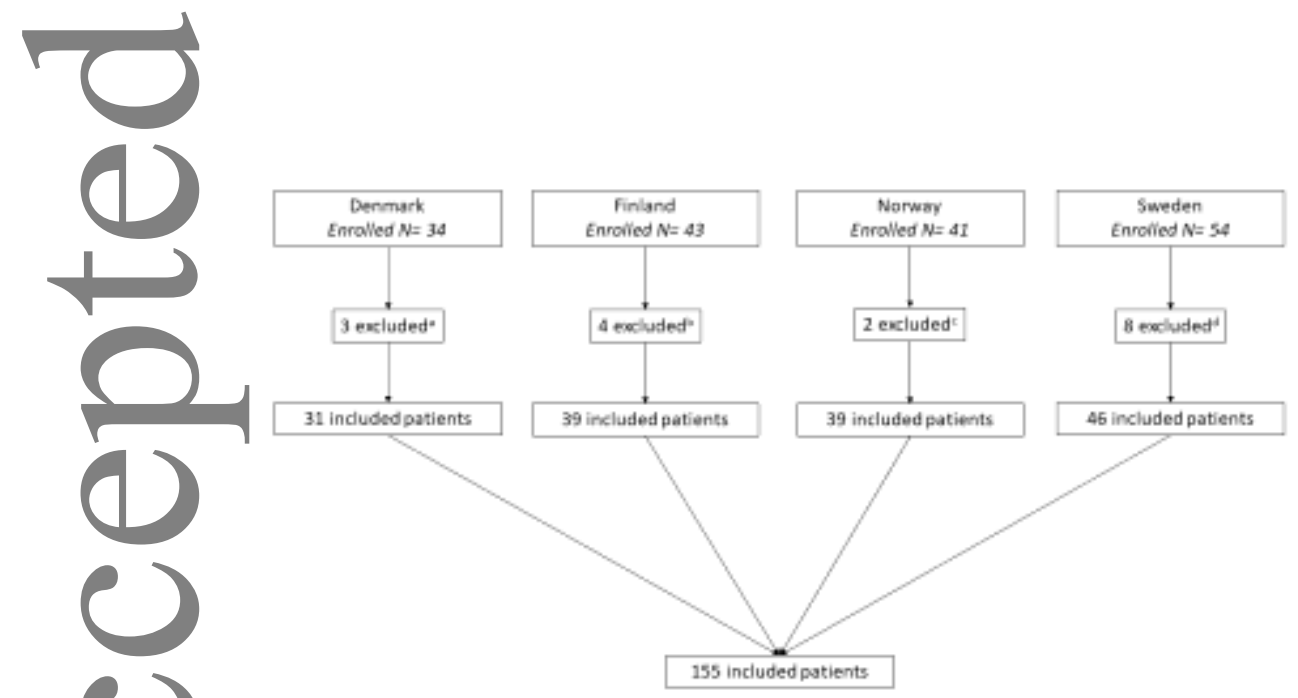